\title{
Fabrication and Characterization of Magnetic Composite Membrane Pressure Sensor
}

\author{
M. A. Khan, A. Alfadhel, J. Kosel \\ Sensing, Magnetism and Microsystems Group \\ King Abdullah University of Science and Technology \\ Thuwal, Saudi Arabia
}

\author{
M. Bakolka \\ Electrical and Computer Engineering Department \\ Effat University \\ Jeddah, Saudi Arabia
}

\begin{abstract}
This paper describes a magnetic field powered pressure sensor, which comprises a coil array and a magnetic composite membrane. The composite membrane is made by embedding a ribbon of the amorphous soft magnetic alloy Vitrovac®, in a $17 \mathrm{~mm}$ x $25 \mathrm{~mm} \times 1.5 \mathrm{~mm}$ Polydimethylsiloxane (PDMS) layer. PDMS is chosen for its low Young's modulus and the amorphous alloy for its high permeability. The membrane is suspended $1.5 \mathrm{~mm}$ above a $17 \times 19$ array of microfabricated planar coils. The coils are fabricated by patterning a $620 \mathrm{~nm}$ thick gold layer. Each coil occupies an area of $36000 \mu^{2}$ and consists of 14 turns. The sensor is tested by subjecting it to pressure and simultaneously exciting it by a $24 \mathrm{~A} / \mathrm{m}, 100 \mathrm{kHz}$ magnetic field. A pressure change from $0 \mathrm{kPa}$ to $5.1 \mathrm{kPa}$, results in a $5400 \mathrm{ppm}$ change in the voltage output.
\end{abstract}

Keywords-Magnetic Composite; Pressure Sensor; Magnetic Amorphous Alloy; Wireless Near Field Sensor.

\section{INTRODUCTION}

Continuous monitoring of pressure is desirable for various applications such as intra-ocular pressure measurement, intracranial pressure measurement, blood pressure measurement and plantar pressure measurement. Multiple sensors have been developed that target these applications [1]-[4]. Wireless operation is a desirable trait for such sensors as it enables noninvasive observation of pressure.

Most commercial pressure sensors available today utilize either piezoelectric, piezoresistive, capacitive or inductive sensing to convert the applied stress into an electrically readable signal. Non-inductive sensors need to be coupled with a planar coil or antenna to enable wireless communication and power transfer, which increases their size, manufacturing complexity and cost [5]. Further some of these sensors use electromagnetic waves for wireless operation [6], which are heavily attenuated by bodily tissues and fluids. This necessitates the use of higher power electromagnetic radiation, which is undesirable.

On the other hand, inductive sensors are capable of native wireless operation through magnetic waves, which are not attenuated by the bodily tissues and fluids. However conventional inductive sensors such as linear variable differential transformer (LVDT) are quite bulky and not suitable for the applications listed above. Most non-inductive sensors are implemented using conventional IC fabrication process, while most inductive sensors use wire wound coils [7], which are difficult to implement using planar processes. According to [8], 2 planar coils facing each other and separated by a small distance can be used as a pressure/displacement sensor, but operation as a pressure sensor has not been presented. Further, packaging 2 dies facing each other in an accurately aligned fashion is quite challenging.

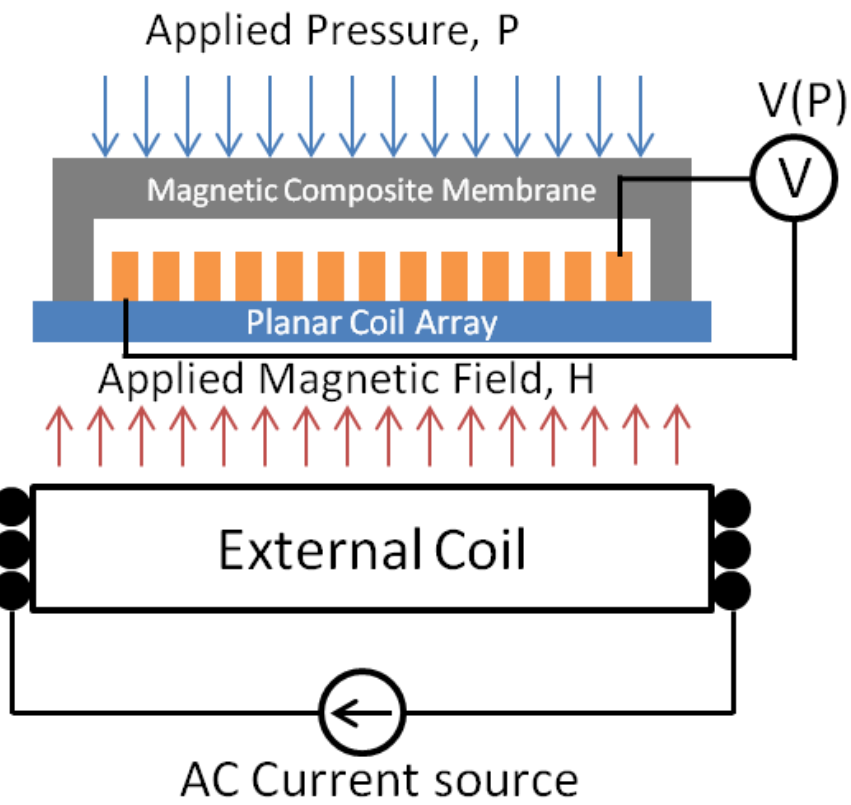

Fig. 1. Schematic of magnetic composite membrane pressure sensor indicating the applied pressure, $\mathrm{P}$, magnetic field, $\mathrm{H}$ and the resultant sensor output voltage, $\mathrm{V}(\mathrm{P})$

In order to overcome these limitations, we propose an inductive pressure sensor that utilizes a planar coil array and a membrane made of high permeability magnetic composite. The magnetic composite membrane consists of an amorphous magnetic alloy ribbon incorporated into a PDMS membrane. The polymer PDMS enables the creation of a membrane with extremely low stiffness coefficient, which exhibits large deflection when subject to pressure. The amorphous magnetic alloy Vitrovac ${ }^{\circledR}$ has been utilized for various sensing applications before [9-12] owing to its soft magnetic properties. It possesses extremely high magnetic permeability [13] and increases the variation of magnetic flux density in the vicinity of the planar coils, due to the deflection of the membrane. 


\section{SENSOR OPERATION}

The implemented sensor consists of a PDMS-amorphous metal composite membrane suspended above a planar coil array. A schematic depiction of the sensor is shown in fig. 1. When a pressure is applied to the membrane, it deflects, changing the magnetic permeability in the vicinity of the coils. For a rectangular membrane with length 1 , width w, stiffness coefficient $\mathrm{k}$, subjected to a force $\mathrm{F}$, the relationship between the applied pressure $\mathrm{P}$ and the resultant displacement $\mathrm{X}$ is given as

$$
P=\frac{F}{w \cdot l}=\frac{k \cdot x}{w \cdot l}
$$

In order to determine the pressure change, the coil is stimulated with an a.c. current, generating an a.c. magnetic field that is affected by the highly permeable ribbon in the membrane. According to Faraday's law of induction the voltage induced in a single turn coil is given as

$$
V(t)=-\frac{d \phi}{d t}=-\mu \cdot A \cdot \frac{d H}{d t},
$$

where $A$ is the area enclosed, $\phi$ is the a.c. flux, $\mu$ is the magnetic permeability of the medium and $\mathrm{H}$ is the magnetic field strength.

The permeability of the medium around the coil, is a function of the deflection of the membrane, which in turn depends on the pressure. Therefore, for a planar coil with $\mathrm{N}$ turns, subject to a monotone magnetic field of frequency $f$ and amplitude $\mathrm{H}_{\mathrm{m}}$, the amplitude of the voltage induced is given as

$$
V_{m}=2 . \pi . f \cdot \mu(P) . H_{m} \cdot \sum_{k=1}^{N} A_{k},
$$

where $A_{k}$ is the area enclosed by the $k^{\text {th }}$ turn of the coil.

For an M-coil array, the amplitude of the induced voltage is given as

$$
V_{m}=2 . \pi . f . H_{m} \cdot \sum_{q=1}^{M}\left[\mu_{q}(P) . \sum_{k=1}^{N} A_{k}\right],
$$

where $\mu_{\mathrm{q}}(\mathrm{P})$ is the magnetic permeability in the vicinity of the $\mathrm{q}^{\text {th }}$ coil. Since the membrane's maximum deflection is at the centre, the coils in this location experience the largest increase in magnetic permeability. Thus, we see that increase in pressure results in a higher permeability of the coils, which in turn increases the induced voltage and vice versa.

\section{FABRICATION}

The sensor fabrication process consists of two major steps: (i) Fabrication of coil array and (ii) Fabrication of magnetic composite membrane and assembly. It is depicted in detail in fig. 2.

\section{A. Coil array fabrication}

The coil array is fabricated using a three layer metal insulator metal process.

We start with a $<100>$ p-type silicon wafer. This wafer is then subject to thermal oxidation to grow a $500 \mathrm{~nm}$ thick

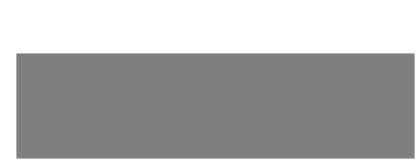

(a)

(c)

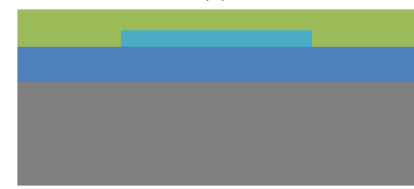

(e)

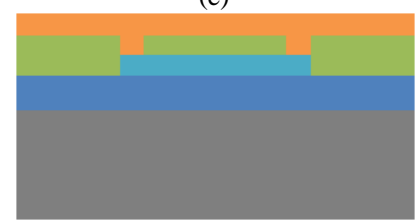

(g)

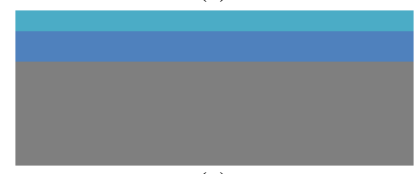

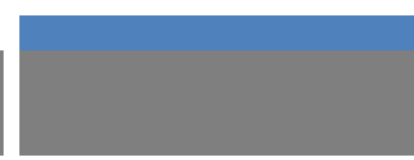

(b)

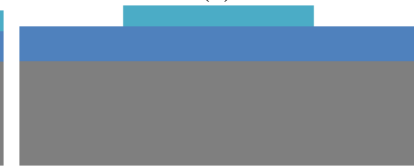

(d)

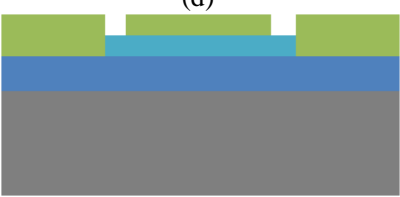

(f)

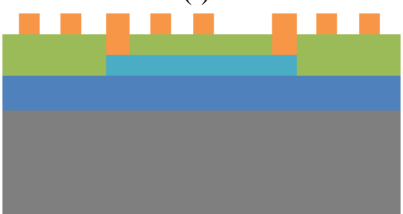

(h)

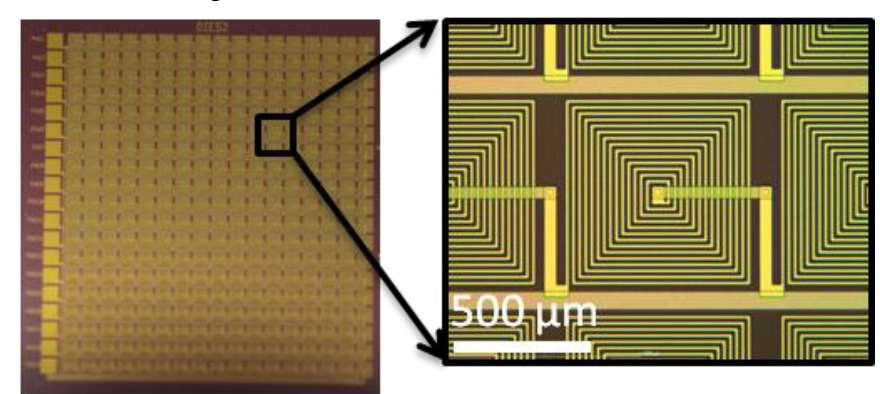

(i)

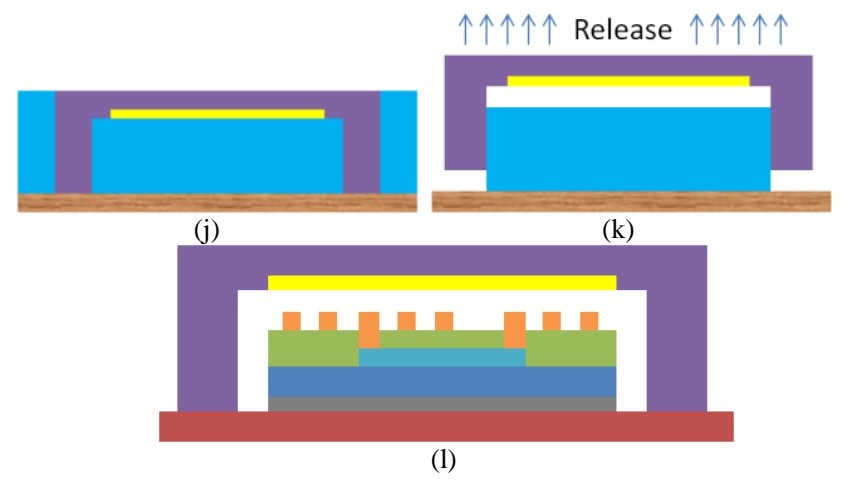

$\square \mathrm{Si} \square \mathrm{SiO}_{2} \square \mathrm{Pt} \square \mathrm{Si}_{3} \mathrm{~N}_{4} \square \mathrm{Au} \square \mathrm{PDMS} \backsim$ Vitrovac PMMA $\mathrm{PCB}$ E Glass

Fig. 2. Fabrication process overview (not drawn to scale) (a) $\mathrm{Si}\langle 100\rangle$ wafer, (b) $\mathrm{SiO}_{2}$ thermal growth, (c) Pt deposition, (d) Lithography and Ar dry etch of Pt, (e) Plasma enhanced chemical vapor deposition silicon nitride deposition, (f) Lithography and nitride etching by reactive ion etching, (g) Au deposition, (h) Lithography and Ar dry etching of Au, (i) Photograph of coil array, (j) Magnetic composite membrane molding, (k) Magnetic composite membrane release, (1) Pressure sensor assembly

silicon dioxide layer, which provides electrical isolation from the semiconductor substrate.

This step is followed by the deposition of a $15 \mathrm{~nm}$ thick layer of titanium using DC sputtering in an argon ambient at 5 mTorr pressure. Without breaking the vacuum, this is followed 
by the deposition of a $120 \mathrm{~nm}$ thick layer of platinum. The titanium layer improves adhesion of platinum to the substrate. A layer of photoresist is spun coated onto the wafer. This is followed by baking and exposure using a mask aligner. The subsequent development step transfers the pattern to the wafer. The patterned wafer is then subjected to Ar dry etching to form the interconnect layer.

Subsequently, a $150 \mathrm{~nm}$ thick layer of silicon nitride is deposited using plasma enhanced chemical vapor deposition (PECVD) at $300^{\circ} \mathrm{C}$. Silane, ammonia and nitrogen gases serve as precursors for this process. The plasma frequency is toggled between high frequency (13.56 MHz) and low frequency (300 $\mathrm{kHz}$ ) states through the process in order to lower the stress inherent in nitride films. Next, photoresist is spun coated, baked, exposed using a mask aligner and developed to transfer the via layer pattern. This is followed by reactive ion etching using sulphur hexafluoride $\left(\mathrm{SF}_{6}\right)$ and trifluoromethane $\left(\mathrm{CHF}_{3}\right)$ gases to create the vias that allow the coil layer to connect with the interconnect layer.

This is followed by the deposition of a $15 \mathrm{~nm}$ thick titanium layer using DC sputtering in an $\mathrm{Ar}$ ambient to promote adhesion. Subsequently, without breaking the vacuum, $620 \mathrm{~nm}$ of $\mathrm{Au}$ are deposited. This is followed by standard mask based photolithography and dry Ar etching, which defines the coil layer.

\section{B. Fabrication of magnetic composite mebrane and assembly}

PDMS (Dow Corning Corp. Slygard $® ~ 184$ ) is prepared by mixing the pre-polymer and the curing agent in a 10:1 ratio by weight. Two pieces of PMMA, cut using a $\mathrm{CO}_{2}$ laser cutter (Universal Laser Systems Inc.PLS 6.75) are used to form the mold, which is used to cast the membrane. The thickness of the inner piece determines the separation between the coil array and the membrane. The length and width of the membrane is the same as the length and width of the inner PMMA piece. The difference in thickness between the outer and inner pieces determines the thickness of the membrane.

The PMMA pieces are bonded to a glass slide using an adhesive. The amorphous metal ribbon is placed on the inner

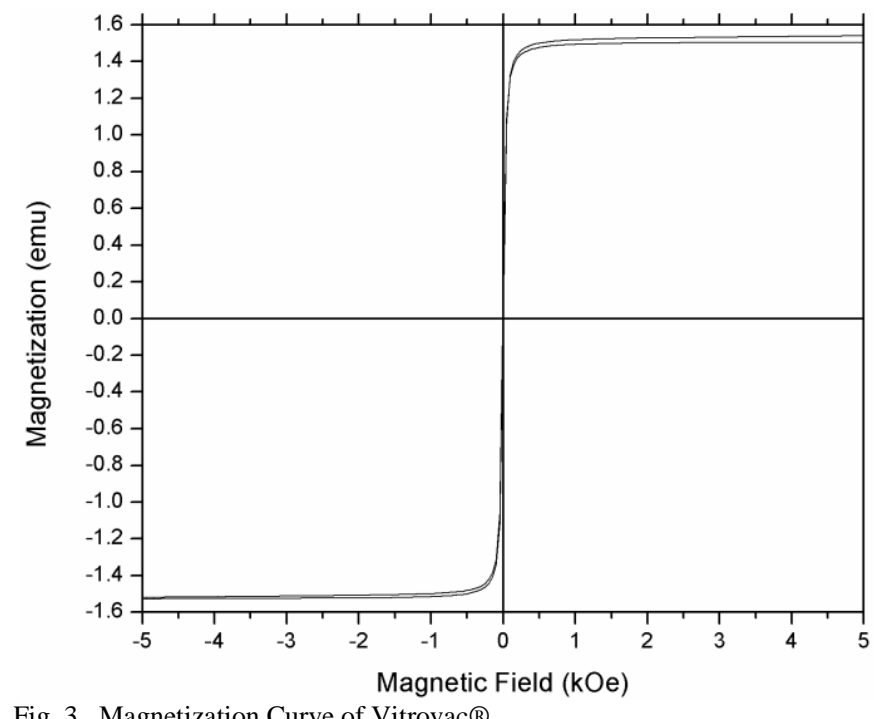

Fig. 3. Magnetization Curve of Vitrovac® mold piece and the PDMS mixture is poured into the mold. The filled mold is subject to vacuum desiccation for 15 minutes, in order to eliminate microbubbles, which helps improve the mechanical properties of the membrane. This is then followed by a baking step at $90^{\circ} \mathrm{C}$ for 60 minutes. The membrane is demolded by carefully removing the outer piece and releasing the membrane.

The coil array is bonded to a PCB using an adhesive. Wirebonding is used to connect the coil array terminals to the PCB pads. The membrane is carefully stuck to the PCB using an adhesive forming the sensor.

\section{RESULTS AND DISCUSSIONS}

\section{A. Magnetic Characteization}

Magnetization curves are obtained for a $4 \mathrm{~cm}^{2}$ piece of the amorphous magnetic alloy at $297 \mathrm{~K}$ using a vibrating sample magnetometer. Silicon grease is used to secure the sample to the sample holder, in order to prevent any movement during measurement. The sample holder is vibrated with an amplitude of $1 \mathrm{~mm}$ and a frequency of $83 \mathrm{~Hz}$, while sweeping the applied magnetic field from $-5 \mathrm{kOe}$ to $5 \mathrm{kOe}$. The resultant magnetization is recorded and the curve obtained is depicted in fig. 3. As expected from a soft magnetic material, the magnetization curve slope is extremely steep at the origin, and the remanent magnetization and coercive field are very low.

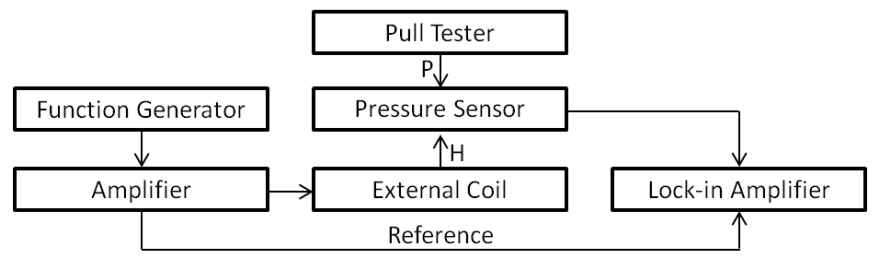

(a)

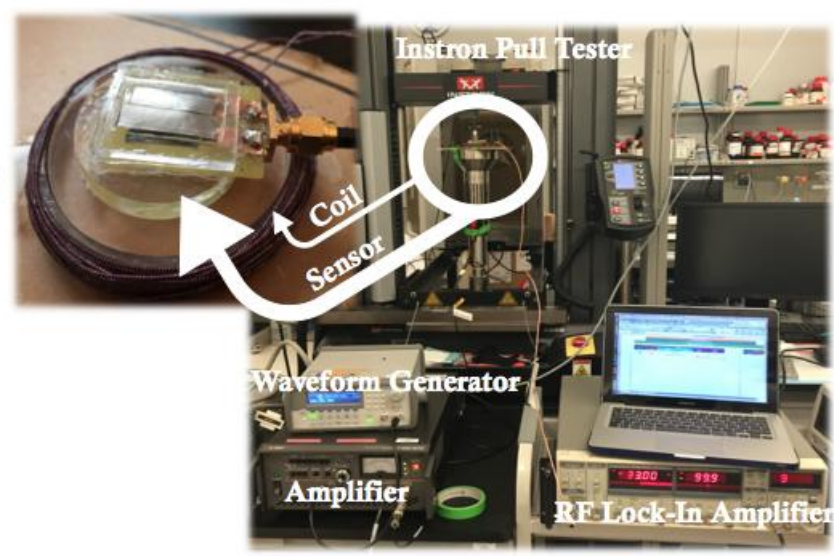

(b)

Fig. 4. (a) Block Diagram of test setup and (b) Actual test setup

\section{B. Sensor Characterization}

A SubMiniature version A (SMA) female connector is soldered to the PCB to read the voltage produced by the sensor coil array. The external coil, which generates the magnetic field for the sensor, is made by winding 30 turns of litz wire on a 6 cm diameter PMMA disc. The sensor is mounted on a $1 \mathrm{~cm}$ thick PMMA disc, which is mounted above the external coil using double sided tape. Thus, the distance between the magnetic field source and the sensor in this experiment is $1 \mathrm{~cm}$. 


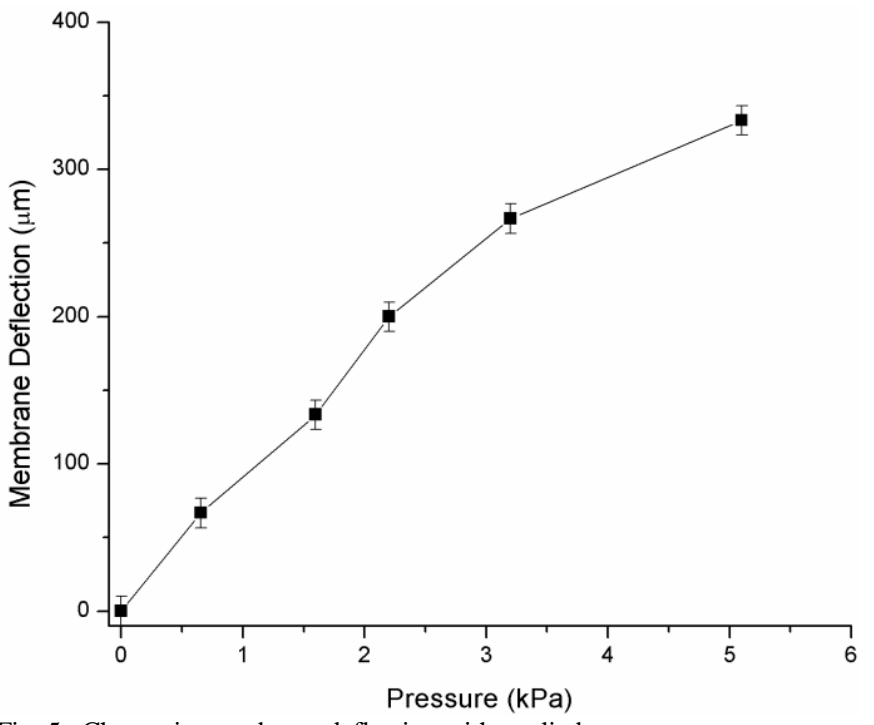

Fig. 5. Change in membrane deflection with applied pressure

A function generator (Agilent 33220A) is used to generate a $100 \mathrm{kHz}$ sinusoidal voltage signal, which is then fed into a high voltage, high current bipolar amplifier (HSA 4012). The gain of the bipolar amplifier is set such that the output signal is a $0.5 \mathrm{~A}, 100 \mathrm{kHz}$ current. This signal drives the stimulating coil. Measurement using Gauss meter indicates a $24 \mathrm{~A} / \mathrm{m}$ magnetic field at a distance of $1 \mathrm{~cm}$ from the stimulating coil.

The setup is mounted on a pull-tester (Instron ${ }^{\circledR}$ 5966), which is modified to use a PMMA head, in order to eliminate eddy current losses, permeability changes that might result in case a metallic head is used. The test setup and the schematic view are given in fig. 4 . The membrane deflection is varied from 0 to $0.33 \mathrm{~mm}$ in steps of $66.67 \mu \mathrm{m}$, while noting the force applied at each step. Dividing the force by the area gives the pressure applied. The resultant voltage signal from the sensor is measured using an RF lock-in amplifier (Stanford Research Systems Inc. SR844), which is supplied with the reference signal from the amplifier. The input impedance of

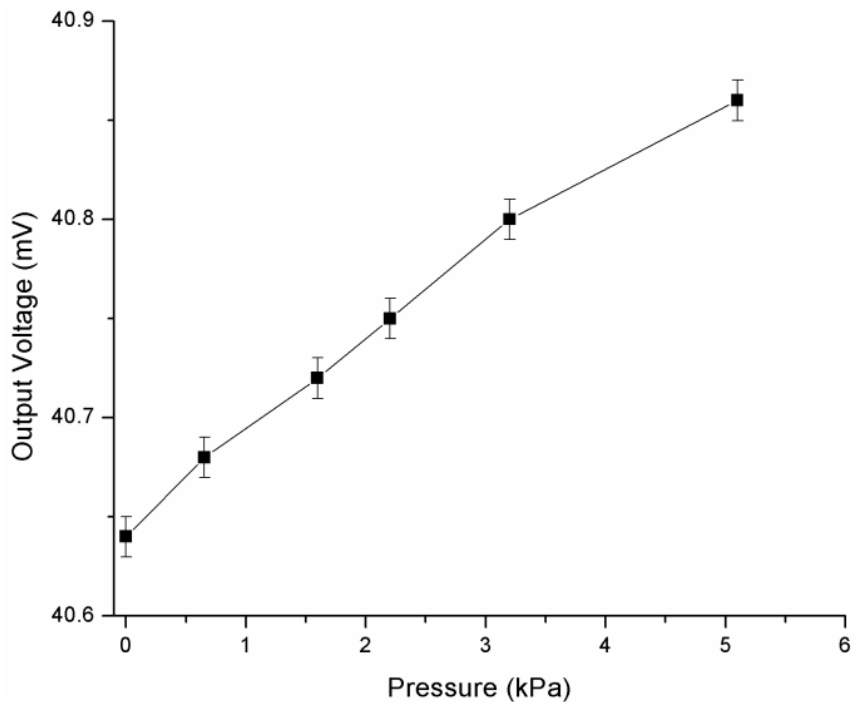

Fig. 6. Sensor output voltage variation with applied pressure the lock-in amplifier is 2 orders of magnitude larger than the internal sensor impedance, thereby reducing the effect of sensor loading.

The resultant pressure versus membrane deflection is plotted in fig. 5, and the pressure versus output voltage curve is depicted in fig. 6. As the applied pressure increases, an increase in membrane deflection and sensor output voltage is observed. The output voltage changes from $40.64 \mathrm{mV}$ to $40.86 \mathrm{mV}$ in response to a change in pressure from $0 \mathrm{~Pa}$ to $5.1 \mathrm{kPa}$. The sensor response is linear in this pressure range with a sensitivity of $43.23 \mu \mathrm{V} / \mathrm{kPa}$. The dynamic range of the lock-in amplifier restricts the resolution of the sensor to $231.3 \mathrm{~Pa}$.

\section{CONCLUSION}

A magnetic field powered pressure sensor capable of measuring pressure ranging from 0 to $5.1 \mathrm{kPa}$ is presented in this paper. The powering field of $24 \mathrm{~A} / \mathrm{m}$ is 5 orders of magnitude lower than the magnetic field used in MRIs. An amorphous soft magnetic alloy ribbon, embedded in PDMS, enhances the magnetic permeability in the vicinity of the sensor coil array, when subject to pressure. The sensor output changes by $220 \mu \mathrm{V}$ in response to a change in pressure from 0 to $5.1 \mathrm{kPa}$, when stimulated by a $24 \mathrm{~A} / \mathrm{m} 100 \mathrm{kHz}$ magnetic field. The sensor has an extremely fine resolution of $231.3 \mathrm{~Pa}$, which is 430 times smaller than the atmospheric pressure. Thus, it is quite capable of measuring most biological phenomena. The sensitivity of the sensor is found to be 1064 $\mathrm{ppm} / \mathrm{kPa}$. By changing the thickness of the membrane and the separation between the coil array and the membrane, the sensitivity and the operational range of the sensor can be tuned for specific applications.

\section{ACKNOWLEDGMENT}

The authors thank Mohammed Y. Alnassar and Ahad Ali for assisting with the coil array fabrication. Mr. Ulrich Buttner helped with the test setup and characterization of the pressure sensor. Research reported in this publication was supported by the King Abdullah University of Science and Technology (KAUST)

\section{REFERENCES}

[1] K. F. Lei, K. F. Lee, M. Y. Lee, "Development of a flexible PDMS capacitive pressure sensor for plantar pressure measurement", Microelectronic Engineering, vol. 99, pp. 1-5, November 2012.

[2] L Rosengren, Y Backlund, T Sjostrom, B Hok and B Svedbergh, " A system for wireless intra-ocular pressure measurements using a silicon micromachined sensor", vol.2, no.3, pp.202-204, 1992.

[3] K.C. Katuri, S. Asrani, M.K. Ramasubramanian, "Intraocular Pressure Monitoring Sensors", IEEE Sensors Journal, vol.8, no.1, pp.12-19, Jan. 2008.

[4] M. Theodor, J. Fiala, D. Ruh, K. Förster, C. Heilmann, F. Beyersdorf, et al., "Implantable accelerometer system for the determination of blood pressure using reflected wave transit time", Sensors and Actuators A: Physical, vol. 206, pp. 151-158, 1 February 2014.

[5] X. Ning, S. P. Chang, J. B. Lee, "A SU-8-Based Microfabricated Implantable Inductively Coupled Passive RF Wireless Intraocular Pressure Sensor", Journal of Microelectromechanical Systems, vol.21, no.6, pp.1338-1346, Dec. 2012.

[6] G. Schimetta, F. Dollinger, R. Weigel, "A wireless pressuremeasurement system using a SAW hybrid sensor", IEEE Transactions 
on Microwave Theory and Techniques, vol.48, no.12, pp.2730-2735, Dec 2000.

[7] E.G. Bakhoum, M.H.M. Cheng, "High-Sensitivity Inductive Pressure Sensor," in Instrumentation and Measurement, IEEE Transactions on , vol.60, no.8, pp.2960-2966, Aug. 2011.

[8] M. B. Coskun, K. Thotahewa, Y.-S. Ying, M. Yuce, A. Neild, and T. Alan, "Nanoscale displacement sensing using microfabricated variableinductance planar coils," Appl. Phys. Lett., vol. 103, no. 14, pp. 143501143504, 2013.

[9] H. Pfützner, E. Kaniusas, J. Kosel, L. Mehnen, T. Meydan, M. Vázquez, M. Rohn, C. Malvicino, B. Marquardt: "Magnetostrictive Bilayers for Multi-functional Sensor Families”. Sensors \& Actuators, 129, 1-2, 154 158 (2006).

[10] J. Kosel, L. Mehnen, E. Kaniusas, H. Pfützner, T. Meydan, M. Vázquez, M. Rohn, A.M. Merlo, B. Marquardt: "Non-Contact Detection of Magnetoelastic Bilayer Position Sensors". Journal of Sensors and Actuators A, 123-124, 349-353 (2005).

[11] E. Kaniusas, H. Pfützner, L. Mehnen, J. Kosel, J.C. Téllez-Blanco, G. Varoneckas, A. Alonderis, T. Meydan, M. Vázquez, M. Rohn, A.M. Merlo, B. Marquard: "Method for continuous non-disturbing monitoring of blood pressure by magnetoelastic skin curvature sensor and ECG". IEEE Sensors Journal, 6, 3; 819 - 828 (2006).

[12] E. Kaniusas, H. Pfutzner, L. Mehnen, J. Kosel, J.C. Téllez-Blanco, E. Mulasalihovic, T. Meydan, M. Vázquez, M. Rohn, C. Malvicino, B. Marquardt: "Optimisation of sensitivity and time constant of thermal sensors based on magnetoelastic amorphous bilayers", Journal of alloys and compounds, 369, 1-2, pp. 198-201 (2004).

[13] G.V. Kurlyandskaya, V.M. Prida, B. Hernando, J.D. Santos, M.L Sánchez, M. Tejedor, "GMI sensitive element based on commercial Vitrovac ${ }^{\circledR}$ amorphous ribbon", Sensors and Actuators A: Physical, Volume 110, Issues 1-3, 1 February 2004, Pages 228-231. 